
$\$$ Research Square
Preprints are preliminary reports that have not undergone peer review.
They should not be considered conclusive, used to inform clinical practice,
or referenced by the media as validated information.

\title{
Evaluation of an effective detection and quantification method for particular microorganisms by comparing NGS-based metagenome profiling data
}

Kyudong Han ( $\nabla$ kyudong.han@gmail.com )

Dankook University - Cheonan Campus https://orcid.org/0000-0001-6791-2408

Jinuk Jeong

Dankook University - Jukjeon Campus: Dankook University

Seyoung Mun

Dankook University - Cheonan Campus

Yunseok Oh

Dankook University - Jukjeon Campus: Dankook University

Chun-Sung Cho

Dankook University College of Medicine

Kyeongeui Yun

HuNBiome

Yongju Ahn

HuNBiome

Won-Hyong Chung

Korea Food Research Institute

Mi Young Lim

Korea Food Research Institute

Tae Soon Hwang

Theragene Bio

\section{Research Article}

Keywords: Metagenome profiling, Quantitative real-time PCR (qRT-PCR), Next-Generation Sequencing (NGS), Genus-specific primers

Posted Date: June 21st, 2021

DOI: https://doi.org/10.21203/rs.3.rs-642362/v1

License: (c) (7) This work is licensed under a Creative Commons Attribution 4.0 International License. Read Full License 


\section{Abstract}

Metagenome profiling research using next-generation sequencing (NGS), a technique widely used to analyze the diversity and composition of microorganisms living in the human body, especially the gastrointestinal tract, has been activated, and there is a growing interest in the quantitative and diagnostic technology for specific microorganisms. According to recent trends, quantitative real-time PCR (qRT-PCR) are still of considerable technique in detecting and quantifying bacteria associated with the human mouth, nasal cavity, and pharynx due to analytical cost and time burden of NGS technology. Here, based on NGS metagenome profiling data produced by utilizing 100 gut microbiota samples, we conducted a comparative analysis of identifying for five bacterial genera proportions (Akkermansia, Bacteroides, Bifidobacterium, Phascolarctobacterium, and Roseburia) within same metagenomic DNA samples through qRT-PCR assay in parallel. Genus-specific primer, targeting the particular gene of each genus for qRT-PCR assay, allowed a statistically consistent quantification pattern with the metagenome profiling data. Furthermore, results of bacterial identification through Sanger validation demonstrated the high genus-specificity of each primer set. Therefore, our study suggests that an approach to quantifying specific microorganisms by applying qRT-PCR method can compensate for the concerns (potential issues) of NGS while also providing efficient benefits to various microbial industries

\section{Key Points}

1) The quantitative real-time PCR (qRT-PCR) is an suitable detection and quantification tool that can counterpart the 16s metagenomics profiling technique.

2) The genus-specific primer designed by investigating a target site (or gene) for a microorganism of interest through large-scale data comparative analysis helps solve the mosaic problem of multiple conserved and variable regions of $16 \mathrm{~s}$ rRNA

3) For bacterial identification through qRT-PCR, nucleotide sequence confirmation via Sanger sequencing is important for designing a genus-specific primer and developing a quantification method.

\section{Introduction}

The high-throughput nucleic acid sequencing technology (Next-Generation Sequencing; NGS) has developed rapidly over the past 15 years, applied to various molecular genetics studies (Gu et al. 2019; Slatko et al. 2018; Stenson et al. 2017). This technology is not only read whole-genome sequences for various species but also widely applied in the molecular diagnostic field, such as prediction of particular human disease through identifying biomarker genes (Okamura et al. 2019; Rim et al. 2017). In addition, advances in NGS technology have recently brought new changes to microbiological research (Cao et al. 2017; Deurenberg et al. 2017; Malla et al. 2018). In past microbiological studies, microbial identification was possible through the conventional Sanger sequencing using bacterial 16S rRNA gene or fungal ITS (Internal Transcribed Spacer) regions only for culture-dependent microorganisms (De Filippis et al. 2018). However, the NGS technology, which can read multiple microbial genomes (be defined as 'metagenome') in specimens, allowed the classification of unculturable microorganisms also (Yarza et al. 2014). This approach to classifying microbiome through metagenome sequencing technology (e.g., wholemetagenome sequencing and 16S rRNA sequencing) has been used to understand complex microbial communities and biological interaction (Feng et al. 2018; Hemmat-Jou et al. 2018). Among the several metagenome sequencing technologies, reading the 16S rRNA gene is generally used as a phylogenetic marker to classify bacterial taxonomy (Theis et al. 2019).

The 16S rRNA gene (approximately $1500 \mathrm{bp}$ ) consists of the nine hyper-variable regions (V1-V9) interspersed among the high-conserved sequences. Because the nine variable regions are a powerful tool that can distinguish the diversity between bacterial strains, the universal primer pairs, including a single or a combination of these regions (e.g., V1V2, V3V4, V4, and V5V6 regions on the 16S rRNA gene), is used for 16S rRNA sequencing (Schriefer et al. 2018; Sperling et al. 2017; Walters et al. 2016). However, there is an issue that reading partial 16S variable regions is difficult to classify between some bacterial species with high-sequence similarity (Gao et al. 2017; Jeong et al. 2021). To overcome this bacterial misclassification problem, some companies (PacBio, Oxford Nanopore, and Loopseq) involved in NGS-based sequencing technology are developing novel metagenome analysis platforms that read the full-length $16 \mathrm{~S}$ rRNA gene to classify microorganisms (Fujiyoshi et al. 2020; Karst et al. 2021; Metras et al. 2020). These developments of continuous metagenome research have led to efforts to establish an association between microbiota (e.g., inhabiting in intestinal mucosa or oral cavity) and human health (Mohajeri et al. 2018; Young 2017). Recently, human gut health has been checked by profiling the gut microbiome composition through feces samples (Abrahamson et al. 2017; Liang et al. 2020; Williams et al. 2019).

The human gastrointestinal tracts are composed of more than 100 trillion different microorganisms such as bacteria, archaea, and viruses, creating a complex microbial ecosystem (Rinninella et al. 2019). For the last ten years, large-scaled gut microbiome studies such as the Human Microbiome Project (HMP) and MetaHIT explained that many human inflammatory diseases, obesity, and neurological diseases are associated with 'dysbiosis' (Gevers et al. 2012; Heiman and Greenway 2016; Nash et al. 2017). The dysbiosis, which is defined to the imbalance of normal microbiota inhabiting the gut, is mainly caused by bad eating habits, stress, and antibiotics. This phenomenon results in an increasing proportion of intestinal harmful bacteria and yeast associated with various human diseases (Baj et al. 2019; DeJong et al. 2020; Losso 2021). In this respect, NGS-based microbial classification has been applied to various microbiome studies, resulting in significant biomedical findings. Still, the metagenome sequencing technology has some issues such as a high-cost burden and timeconsuming if the purpose is intended to detect just specific microorganisms (O'Sullivan et al. 2013). For these reasons, the real-time PCR (also denoted as quantitative real-time PCR-qRT-PCR) is usually used to confirm rapid microbial detection through target gene quantification from clinical samples taken from participants (Pereira et al. 2017; Wang et al. 2011). Indeed, many companies and researchers involved in microbial molecular diagnosis are attempting to overcome the issue of high-sequence similarity between bacterial species arising from the 16S rRNA gene by developing specific primer pairs for using qRTPCR equipment.

In this study, we investigated the concordance between qRT-PCR and NGS technology to confirm the efficiency of microbial quantification for genus level associated with the human health. Since NGS is currently not suitable as a method for identifying species-level microorganisms, we have confirmed the 
similarity of the two technologies at the genus level. Based on NGS metagenome profiling data produced by utilizing 100 gut microbiota samples, we conducted a comparative analysis of quantification accuracy for five bacterial genera (Akkermansia, Bacteroides, Bifidobacterium, Phascolarctobacterium, and Roseburia) through qRT-PCR assay in parallel. Genus-specific primer targeting the particular gene (such as transcription termination/anti-termination protein; nusG) with one of the genera allows relatively similar quantification results with the 16S V3-V4 metagenome profiling data. Additionally, we crossvalidated the genus-specificity of the designed target primers via Sanger sequencing with qRT-PCR products as template DNA.

\section{Materials And Methods}

\section{1) Human stool samples collection}

One hundred human stool samples were collected using the OMNlgene•GUT stool swab kit (DNAgenotek, Canada) from healthy adult men and women participating in the Korean Gut Microbiome Database Project of the Korea Food Research Institute (KFRI, South Korea). All stool samples were then stored at $-80^{\circ} \mathrm{C}$ for the experimental downstream processing. The sampling was carried out with the prior informed consent of all participants before this study began. None of participants affected the research results, such as taking medication before the study began. The clinical sample collection in this study (stool samples collection) was approved by the ethics committee of Theragen Bio (Theragen Bio, South Korea) Institutional Review Board (IRB Protocol Number: 700062-20180905-JR-005-01). All clinical experiments applied to this study were carried out according to the guidelines and regulations of the declaration of Helsinki.

\section{2) Metagenomic DNA extraction}

Total metagenomic DNA (mDNA) from 100 stool samples was isolated using the QIAamp DNA Stool Mini Kit (Qiagen, Germany), and all experimental processes were performed in accordance with the optimal protocols provided on the DNA extraction kit. The quality check of all isolated mDNA was conducted using a Bioanalyzer (Agilent 2100, USA) at the Center for Bio-medical Engineering Core Facility (Dankook University, South Korea). All mDNA samples were then stored at $4^{\circ} \mathrm{C}$ until the following process.

\section{3) IIIumina 16S V3-V4 amplicon sequencing library preparation and sequencing}

A total of 100 metagenome sequencing libraries were prepared according to the Illumina $16 \mathrm{~S}$ amplicon sequencing library construction workflow (Illumina, USA). The Illumina platform targeted an area containing the V3-V4 hyper-variable region of the bacterial 16S rRNA gene. The PCR amplification of the target region was started immediately after the mDNA was extracted. The 16S V3-V4 amplicon was amplified using KAPA HiFi Hot Start Ready Mix (2x) (Roche, Germany). For this purpose, a pair of V3-V4 target-specific universal primers recommended by Illumina were used. The primer sequences were as follows:

16S 341F forward primer is 5' -TCGTCGGCAGCGTCAGATGTGTATAAGAGACAGCCTACGGGNGGCWGCAG-3' and 16S 806R reverse primer is 5' GTCTCGTGGGCTCGGAGATGTGTATAAGAGACAGGACTACHVGGGTATCTAATCC-3'. After the PCR amplification, the clean-up process of all PCR products was conducted using the AMPure XP beads (Beckman Coulter, USA). And then, additional PCR amplification was performed to add multiplexing indices and Illumina sequencing adapters using the Nextera XT Index Kit (Illumina, USA). The final PCR products were then purified once again using the AMPure XP beads. After the amplicon library construction, the $16 \mathrm{~S}$ metagenome sequencing was carried out using the paired-end $2 \times 300 \mathrm{bp}$ Illumina MiSeq protocol (Illumina MiSeq, USA; (Wu et al. 2016)).

\section{4) Bacterial genus-specific primer design methods}

Genus-specific primers for qRT-PCR amplification were designed to identify and absolute quantify the particular five bacterial genera from mDNA. The overall process for primer design in this study is as follows.

1. Bacterial genera and target gene selection; Based on 16S V3-V4 metagenome sequencing profiling data, five human intestinal bacteria at the genus level associated with health or disease were listed. Particular genes to detect each bacterial genus through the qRT-PCR were then selected.

2. Sorting of NCBI annotation information; The National Center for Biotechnology Information Database (NCBI DB) was used to obtain sequence information about the selected genes to target each bacterial genus. First, the 'Identical Protein Groups' category was used to extract a comprehensive summary table that explains the gene annotation information on the NCBI DB (e.g., nucleotide and protein accession number, organism at species and strain level, etc.). Next, the ambiguously classified information on the table, such as 'hypothetical protein', was excluded from the list. Then, repetitive information about the randomly selected bacterial species (included strain level) belonging to each genus was filtered. Finally, all 'Protein ID' on the filtered list were isolated to obtain the amino acid sequence information needed to convert to gene sequence.

3. Extraction of coding sequence information; The Batch Entrez open web bioinformatics tool linked with NCBI DB (www.ncbi.nlm.nih.gov/sites/batchentrez) was used to convert amino acid information about specific genes into coding sequences (CDS) in the form of the FASTA format.

4. Multiple sequence alignment and selection of target-specific regions; To find the consistent sequence regions for qRT-PCR primer design, the multiple sequence alignment of the CDS information was performed using the BioEdit 7.2v software.

5. In silico test; The experimental suitability (Tm value, GC\%, and potential for primer-dimer to form, etc.) for qRT-PCR of the selected primer pairs were checked using the Oligo calc and Oligo Analysis open web tool (http:// biotools.nubic.northwestern.edu/; http://www.operon.com/tools/oligo-analysistool.aspx). Next, the NCBI Nucleotide BLAST (https://blast.ncbi.nlm.nih.gov) was used to confirm the primer specificity for the targeted bacterial genus.

5) Bacterial quantification using qRT-PCR 
The qRT-PCR was conducted to quantify the five bacterial genera frequencies from the isolated 100 mDNA samples. First of all, double-stranded DNA concentration within all mDNA samples was measured using the Qbit Fluorometer $4.0 \mathrm{v}$ at the Center for Bio-medical Engineering Core Facility (Dankook University, South Korea) and 1X dsDNA HS Assay kit (ThermoFisher Scientific, USA). Then, all template mDNA was then normalized to an identical concentration ( $10 \mathrm{ng} / \mathrm{ul}$ ) via dilution by using distilled water. Next, a standard curve for 100 diluted DNA samples was calculated to confirm that the template DNA concentration used in qRT-PCR was consistently normalized. In this step, the cycle threshold (Ct) value for amplicon quantity in qRT-PCR measured using the bacterial 16S V4 primer pair (515F and 806R) was applied. These Ct values were reflected from the 10 -fold serial dilution (10-1, $10^{-2}$, and $10^{-3}$ dilution) samples for each formerly normalized mDNA sample. Finally, the bacterial genera frequency within 100 template mDNA was confirmed using the StepOnePlus ${ }^{\text {TM }}$ Real-Time PCR (ThermoFisher Scientific, USA) at the Center for Bio-medical Engineering Core Facility (Dankook University, South Korea) and QuantiSpeed SYBR No-Rox kit (PhileKorea, South Korea). Each qRT-PCR primer annealing temperature condition applied at this step is as follows; Akkermansia: $65^{\circ} \mathrm{C}$, Bacteroides: $65^{\circ} \mathrm{C}$, Bifidobacterium: $62.5^{\circ} \mathrm{C}$, Phascolarctobacterium: $62^{\circ} \mathrm{C}$, and Roseburia: $65^{\circ} \mathrm{C}$. Each summary table $($ Supplemental Table S6), which included the $\mathrm{Ct}$ value of five bacterial genera, was used to compare NGS-based bacterial frequency data.

\section{6) Sanger sequencing}

The Sanger sequencing was conducted to verify the molecular specificity and experimental accuracy of the five primer pairs designed for this study. First of all, the ten qRT-PCR amplicon samples (Top five and bottom five of the measured Ct value) were sorted from each qRT-PCR summary table (Supplemental

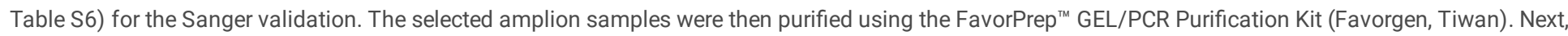
molecular cloning through ligation and transformation process were carried out using TOPcloner ${ }^{\text {TM }}$ TA Kit (Enzynomics, South Korea) and DH5a chemically competent E. coli (Enzynomics, South Korea) to obtain template DNA containing amplicon sequence. A colony PCR amplification using the M13 primer pair (M13F: 5'-GTAAAACGACGGCCAG-3'; M13R: 5'-CAGGAAACAGCTATGAC-3') was conducted to check whether the target sequence was inserted in the plasmid cloning vector. The colony PCR products were once again purified through the FavorPrep ${ }^{\text {TM }}$ GEL/PCR Purification Kit (Favorgen, Tiwan). The Sanger sequencing with purified products as template DNA was carried out using the ABI 3500XL Genetic Analyzer sequencer (ThermoFisher Scientific, USA) at the Center for Bio-medical Engineering Core Facility (Dankook University, South Korea). The ABI and FASTA format data generated after sequencing were used as input data in the NCBI Nucleotide BLAST tool for bacterial genus identification.

\section{7) 16S V3-V4 data processing and microbial community analysis}

The 16S V3-V4 sequencing reads were demultiplexed using the split_libraries_fastq.py function in QIIME2 metagenome analysis pipeline and sequences were quality trimmed using the Divisive Amplicon Denoising Algorithm 2 (DADA2) pipeline in $\mathrm{R}$ (version 3.3.2) with the parameters; $\mathrm{EE}=2$, TruncL= $\mathrm{c}(200,180$ ) and $q=10$. The set of unique $16 S$ V3-V4 DNA sequences, referred to as amplicon sequence variants (ASVs), were then inferred using DADA2 and an ASV table of read counts per ASV per sample was generated. ASV taxonomy were taxonomically classified using the sklearn-based Naive Bayes classifier with the SILVA 138v 16S rRNA database.

\section{8) Statistical analysis}

An association analysis of the bacterial genera proportions data within 100 samples, measured from the qRT-PCR assay and $16 \mathrm{~S}$ metagenome sequencing results, was conducted through a statistical test using the Spearman correlation test (Fig. 4; Table 3). The Spearman correlation's statistical significance evaluated the statistical similarity between the two methods was denoted as asterisk $(*)$ if $P$-value $<0.05$ and $\mathrm{R}$ value (Spearman's value) $>0.5$.

\section{Results}

\section{1) Selection of five bacterial genera from 165 metagenome analysis data}

This study confirmed the consistency of the relative proportion pattern within the samples of five particular bacterial genera between the 16S metagenome profiling data and qRT-PCR quantitative results (Fig. 1). We collected human stool specimens from 100 healthy Korean (adult men and women). Then, we extracted mDNA from the 100 specimens and simultaneously checked the DNA quality (e.g., DNA degradation, concentration, and purity) for accurate microbial quantification. To select bacterial genus for quantifying frequency in the specimens, we successfully prepared the Illumina $16 \mathrm{~S}$ V3-V4 short-read amplicon sequencing libraries using the 100 mDNA samples, and NGS-based 16S metagenome sequencing was performed. As a result of $16 \mathrm{~S}$ metagenome sequencing, the average demultiplexed reads count generated from 100 samples was 133,288, of which 65,892 were filtered through the DADA2 pipeline with non-chimeric reads. (Supplemental Table S1). After 16S V3-V4 sequencing data processing, the total number of bacterial ASV taxonomy classified from the SILVA 138v 16S rRNA gene reference database was 6,902 (with more $70 \%$ classification confidence threshold), of which 338 were classified at the genus level (Supplemental Table S2). Based on the ASV taxonomy classification results at the genus level, we selected the Akkermansia, Bacteroides, Bifidobacterium, Phascolarctobacterium, and Roseburia. These five genera are the composition of human gut microbiota and are probiotic bacterial strains closely related to obesity, dysbiosis, and Inflammatory Bowel Disease prevention. (Gomez-Gallego et al. 2016; John and Mullin 2016; Liu et al. 2018; Russell et al. 2011; Wang et al. 2020). They are also beneficial bacteria that contribute significantly to the production of the short-chain fatty acid (SCFA; e.g., acetic, butyric, and propionic acid), which is key to human health control, such as increasing immunity, maintaining intestinal homeostasis, and preventing fat accumulation in the body (Markowiak-Kopec and Slizewska 2020). Considering these interesting bacterial features, we applied these five selected genera to compare the relative proportions of particular bacterial load within the samples, measured using the qRT-PCR assay and NGS frequency data.

\section{2) Bacterial genus-specific primer design}

We selected the five bacterial taxa (Akkermansia, Bacteroides, Bifidobacterium, Phascolarctobacterium, and Roseburia) at the genus level based on the formerly performed $16 \mathrm{~S}$ metagenome profiling data. To quantify the bacterial load within the samples using the qRT-PCR assay, we designed genus-specific 
primer pairs to detect these five genera (Table 1). Considered that the high-sequence similarity of the 16S rRNA gene internal hyper-variable region would be an obstacle to detecting the specific genus, we used sequence information on certain bacterial gene domains annotated on the NCBI reference database (Supplemental Table S3; show detailed in the Materials and Methods section). First of all, we applied the sequence information of nus $G$ gene, which encodes a transcription termination/antitermination protein essential for bacterial transcription (Lawson et al. 2018). For the Bifidobacterium and Akkermansia, it was challenging to find consistent regions in the multiple sequence alignment results between the bacterial species belonging to the genus. Therefore, we reselected the transaldolase and D-alanine D-alanine ligase $(d d l)$ gene sequences as a target-specific gene for Bifidobacterium and Akkermansia. In the case of Bifidobacterium, we referred to previous studies that Bifidobacterium species has a gene coding region capable of expressing at least 14 types of transaldolase that can be differentiated using the protein electrophoresis method (Requena et al. 2002). Additionally, we selected the ddl gene as a target gene of Akkermansia, considering that it is an essential factor for bacterial cell wall synthesis (Ellsworth et al. 1996). Finally, we conducted an in silico test using the NCBI Nucleotide BLAST web tool to validate the bacterial genus-specificity of five primer pairs designed for this study (Supplemental Fig. S1). Although some in silico test results showed classification results for microorganisms other than the targeted bacterial genus (e.g., Raphanus sativus, Rodentibacter pneumotropicus, and Acetobacterium woodii), we determined that it would not affect our qRT-PCR assay because they were eukaryotic or not human gut bacteria (Banihani 2017; Fingas et al. 2019; Bache and Pfenning 1981).

\section{3) Quantification and Normalization of metagenomic DNA}

Before estimating a particular bacterial load within the human fecal samples, we normalized the bacterial DNA concentration, which was included in the 100 mDNA samples, through the standard curve calculation using the qRT-PCR (Fig. 2; Table 2; Supplemental Table S4). We used 10-fold serial diluted mDNA samples $\left(10^{-1}, 10^{-2}\right.$, and $10^{-3}$ dilution from $10 \mathrm{ng} / \mathrm{ul}$ concentration of dsDNA per each sample) and bacterial $16 \mathrm{~S}$ V4 universal primer (515F and $\left.806 \mathrm{R}\right)$ for this qRT-PCR assay. Theoretically, considering that the $16 \mathrm{~S}$ rRNA gene is the most conserved region on the bacterial genome, we have determined that it is appropriate to apply the 16S V4 specific-primer pair to the internal bacterial genomic DNA quantification of the mDNA samples (Frank et al. 2008; Tran et al. 2017). As a result of the qRT-PCR assay, we confirmed that the average Ct values about all $10^{-1}, 10^{-2}$, and $10^{-3}$ diluted samples for the V4 amplification were measured at $27.31,23.26$, and 19.85 , respectively. In addition, we could also confirm that the standard deviation (SD) and the coefficient of variation (CV) value of each $\mathrm{Ct}$ value for the three dilution factors were averaged 0.55 and 2.41 percent. Calculating the standard curve based on these measurements, the $\mathrm{R}^{2}$ value of the trend line connecting Ct values for all 10 -fold diluted samples was about 0.97 . Additionally, we found that the $R^{2}$ value of the trend line was close to 1 when calculating the standard curve based on the average Ct value. These results showed that our systematic quantification challenge for the particular genera was conducted with the bacterial DNA samples measured and normalized by comprehensive calculation methods.

\section{4) Parallel comparison of qRT-PCR and $16 S$ metagenome profiling data}

We compared the relative bacterial proportion similarity of the five genera within the human gut microbial community between the qRT-PCR assay using formerly normalized 100 mDNA samples (10 ng/ul per sample) and the NGS-based 16S metagenome profiling data (Fig. 3; Fig. 4; Table 3; Supplemental Table S5; Supplemental Table S6). In order to this comparative approach, we calculated the potential bacterial frequency (1/2 $\mathrm{Ct}$ value $)$ and proportion of each genus within the 100 samples using the Ct value exported by qRT-PCR assay. The multiple comparison data in the Fig. 3 showed that relative distribution of the five bacterial genera proportions within each sample was visualized by the bacterial abundance bar plot and cumulative curve graph. Comparing the qRT-PCR assay with NGS proportions data in parallel, we found that the relative proportion patterns of the five bacterial genera were remarkably consistent between the two different quantification methods. We highlight that four bacterial (Akkermansia, Bifidobacterium, Phascolarctobacterium, and Roseburia) genus present in low proportions in 100 samples of human gut microbiota also confirmed significant relative proportions of the quantification results. Additionally, we verified an association between bacterial proportions data measured from two different quantification methods using the Spearman correlation statistical analysis to supplement these parallel comparison results. As the statistical analysis result, we could confirm that the Spearman correlation values for all bacterial genus groups were significantly calculated (P-value $<0.05$; Spearman's rho value $>0.5$ ). Considering that the Spearman analysis evaluates statistically positive similarity if the correction value (Spearman's rho value) is 0.4 or higher, we could confirm the significant similarity of the relative bacterial proportion measured between the NGS and qRT-PCR assay (Ramsey 1989). Thus, our comparative results showed that the five genus-specific primer pairs have a high-binding sensitivity and specificity for the target mDNA.

\section{5) Verification of primer specificity}

We performed Sanger sequencing of the qRT-PCR amplicon products to confirm the of the bacterial genus-specificity of five primer pairs designed for this study, and verified the sequencing results by BLAST (Basic Local Alignment Search Tool) (Fig. 5; Table 4; Supplemental Table S7). First, we conducted a ligation process to insert qRT-PCR amplicon products with top 5 and bottom $5 \mathrm{Ct}$ values measured per each bacterial genus-group as a TOPO TA cloning plasmid vector for transformation. We applied the TOPO TA vector with M13 primer regions (M13F and M13R; approximately 200 bp) because the input library length for high-quality Sanger sequencing results is at least $400 \mathrm{bp}$ (Kchouk et al. 2017). After the transformation process, we isolated five white colonies (potentially transformed component cells) per each LB culture medium and performed direct colony PCR using the M13 primers. Next, we performed the Sanger sequencing to confirm the sequence information of qRT-PCR amplicon. When the Sanger sequencing results were confirmed through the BLAST search using the blastn parameter, we found that the taxonomy definition rate with the NCBI DB was almost 100\% in the four groups (high and low proportion groups of the Akkermansia, BacteroidesPhascolarctobacterium, and Roseburia). For the Bifidobacterium, the bacterial taxonomy definition rates of high and low groups were $92 \%$, and $60 \%$, respectively. Although some BLAST results of the Bifidobacterium_low groups were resulted to be undefined with NCBI DB, we could find that the relative proportion for 100 people calculated from the NGS frequency and qRT-PCR Ct value was almost similar. As a result of Sanger validation, the qRT-PCR and NGS proportion data shown in Supplemental Table S6 of the Bifidobacterium_low group samples for which the NCBI nucleotide BLAST search was not valid (less than $60 \%$ of bacterial taxonomy definition rate per each plate) were as follows; Bif_Mi_01 (Candidate 50): 0.01 and 1.80, Bif_Mi_02 (Candidate 77): 0.01 and 0.00, Bif_Mi_03 (Candidate 83): 0.01 and 0.00. In this regard, this issue found at the Bifidobacterium_low group was likely to result in low transformation efficiency in the TA cloning process due to i) the too low bacterial frequency within the samples and ii) high $\mathrm{G}+\mathrm{C}$ content 
(approximately $63 \mathrm{~mol} \%$ ) in the transaldolase gene coding region of the Bifidobacterium genome, rather than misbinding of the specific primer pairs that occurred during the qRT-PCR amplification (Freitas and Hill 2018; Park et al. 2019; Serafini et al. 2012). Considering that identification of the target bacterial genus was possible in all Sanger validation results of the selected genus groups reflecting the high and low genus proportions within each sample, we confirmed that the five specific primer pairs designed for this study are sufficient to be applied to bacterial detection and quantification using the qRT-PCR assay.

\section{Discussion}

Since the Human Genome Project, high-throughput sequencing-based microbiome research projects such as the HMP and MetaHIT consortium have emphasized the importance of identifying the association between microorganisms and human diseases. In particular, these projects explained that the causes of various physical diseases are closely related to the "dysbiosis" phenomenon, in which normal intestinal microbiota is unbalanced (Consortium 2010; Gevers et al. 2012). Accordingly, various probiotic therapy-associated industries and researchers have classified the human gut microbial composition using NGS technology to identify the relative intestinal proportions of beneficial and harmful bacteria and their biological effects (Sharifi-Rad et al. 2020; Shukla 2017). However, NGS technology's high analytical cost burden and time consumption are considered important issues in microbiome-associated health care industries. Furthermore, the microbial misclassification problem at the species level caused by the high sequence similarity within the 16S rRNA gene is also regarded as a challenge to be addressed (Callahan et al. 2021; Jeong et al. 2021). Therefore, some researchers have suggested that running experiments in parallel with qRT-PCR, which enables accurate and rapid detection and quantification of the target genes, is a solution to compensate for these issues of the NGS technology. Because the high fluorescence sensitivity of the equipment enables accurate detection of specific microbial species in the sample, the qRTPCR assay is still used as a 'gold standard method', especially in the molecular microbial diagnostics and probiotics fields (Masco et al. 2007; Shehata et al. 2019).

Through parallel comparison analysis, we evaluated that the qRT-PCR technique represents an excellent alternative to existing NGS-based 16S V3-V4 metagenome sequencing methods as it enables reliable detection and quantification (for the gut-associated microbiota: Akkermansia, Bacteroides, Bifidobacterium, Phascolarctobacterium, and Roseburia). Since quantifying the gut microbiome composition of healthy subjects is necessary to establish a baseline against which microbiome changes can be detected in pathological conditions, we performed normalization to the initial microbial density in the V3V4 hyper-variable region of the 16S rRNA gene. We minimize errors in specific microbial populations through this process by quantifying the total load of internal standards and 16S rRNA genes in the meta-samples (Tran et al. 2017). The process of absolute quantification of internal standards as well as in bacterial quantitation systems utilizing qRT-PCR will avoid sacrificing high rates due to abundance and amplification bias in amplicon sequencing that can occur in NGS-based metagenome sequencing and is essential to maintain reproducibility (Zemb et al. 2020). Although the 16S rRNA gene primer is specifically designed and optimized for qRT-PCR or NGS, it is recommended to consider the potential bias and the miss annealing due to sequence similarity between specific bacterial strains for qRT-PCR. In our study, the qRT-PCR primers target CDS sites of a gene (e.g., bacterial housekeeping genes such as nusG or $d d l$ ) representing a particular microbial genus to ensure high sensitivity and reduce biased quantitative results by sequence similarity. In addition, to evaluate the accuracy and sensitivity of the designed genus-specific primer, we selected five amplicon samples with the high and low frequency generated after qRT-PCR amplification, respectively, and confirmed Sanger sequencing validation. As a result, almost all samples where genus-specifically amplified amplicons were generated, suggesting how specific our target primers are and how respectable they are for particular genus quantification. We assumed that several undefined samples through Sanger sequencing validation might not be identified due to the reduced PCR cloning efficiency with the high $\mathrm{G}+\mathrm{C}$ contents of the target CDS or the minimal DNA content of the Bifidobacterium. Nonetheless, the evaluation of the specificity and sensitivity of primers used for the quantification of microorganisms using Sanger sequencing validation suggests that it is an essential task as a standard research procedure for clinical diagnostic research and industrial development. As a result of cross-checking the correlation of the measured bacterial proportions between two different quantification methods through Spearman correlation statistical analysis, it was also verified that the qRT-PCR method was sufficient and accurate for quantitative analysis of specific microbial genera in meta-samples. Therefore, we propose an efficient system in fields requiring rapid quantification of indicator microorganisms required in medicine, agriculture, marine biology, and etc.

In conclusion, we confirmed through a parallel comparative analysis with $16 \mathrm{~S}$ metagenome sequencing data that the qRT-PCR method can complement some issues (such as high analytic cost burden or time consumption) of the NGS technology, which applies to various microbial industries. Our findings showed that the relative bacterial proportions pattern of the five genera within samples, measured via the qRT-PCR assay performed under normalized mDNA concentration conditions, was statistically similar to $16 \mathrm{~S}$ metagenome sequencing data. Additionally, we cross-validated bacterial genus-specificity of the five primer pairs designed for the qRT-PCR assay through the Sanger sequencing and NCBI BLAST test for bacterial identification. In this respect, we affirmed that a qRT-PCR method is one of appropriate tools for identifying the relative proportions of particular microorganisms within the sample. Furthermore, we suggest that applying qRT-PCR to specific microbial validation in the NGS-based microbial diagnosis industry can compensate for the concerns of NGS technology by providing economical, fast, and accurate services to consumers in terms of turnaround time.

\section{Declarations}

\section{Author's contributions}

K.H. designed and supervised the project; C.C., S.M., Y.O. and J.J. performed experiments; K.Y., T.H., W.C., M.L., A.Y., S.M., and J.J. analyzed the data; Y.A., K.H., S.M., and J.J. wrote the manuscript. All authors read and approved the final manuscript.

\section{Funding}

No funding was received to assist with the preparation of this manuscript. 


\section{Data Availability}

The datasets generated during the current study are included in this published article and its supplementary information file and are available from the corresponding author on reasonable request.

\section{Ethical approval}

All clinical samples (stool samples) were collected from healthy adult men and women participating in the Korean Gut Microbiome Database Project of the Korea Food Research Institute (KFRI, South Korea). The human experiment in this study (samples collection) was approved by the ethics committee of Theragen Bio (Theragen Bio, South Korea) Institutional Review Board (IRB Protocol Number: 700062-20180905-JR-005-01)

\section{Conflicts of interest}

The authors have no conflicts of interest to declare that are relevant to the content of this article.

\section{Consent to participate}

Not applicable.

\section{Consent to publication}

Not applicable.

\section{Acknowledgements}

The authors gratefully acknowledge the Center for Bio-Medical Engineering Core Facility at Dankook University for providing critical reagents and research equipment.

\section{References}

Abrahamson M, Hooker E, Ajami NJ, Petrosino JF, Orwoll ES (2017) Successful collection of stool samples for microbiome analyses from a large communitybased population of elderly men. Contemp Clin Trials Commun 7:158-162 doi:10.1016/j.conctc.2017.07.002

Bache R, Pfenning N (1981) Selective Isolation of AceWbacterium woodii on Methoxylated Aromatic Acids and Determination of Growth Yields. Archives of Microbiology 130:255-261 doi:https://doi.org/10.1007/BF00459530

Baj A, Moro E, Bistoletti M, Orlandi V, Crema F, Giaroni C (2019) Glutamatergic Signaling Along The Microbiota-Gut-Brain Axis. Int J Mol Sci 20(6) doi:10.3390/ijms20061482

Banihani SA (2017) Radish (Raphanus sativus) and Diabetes. Nutrients 9(9) doi:10.3390/nu9091014

Callahan BJ, Grinevich D, Thakur S, Balamotis MA, Yehezkel TB (2021) Ultra-accurate microbial amplicon sequencing with synthetic long reads. Microbiome 9(1):130 doi:10.1186/s40168-021-01072-3

Cao Y, Fanning S, Proos S, Jordan K, Srikumar S (2017) A Review on the Applications of Next Generation Sequencing Technologies as Applied to Food-Related Microbiome Studies. Front Microbiol 8:1829 doi:10.3389/fmicb.2017.01829

Consortium SDEaTM (2010) MetaHIT: The European Union Project on Metagenomics of the Human Intestinal Tract. Metagenomics of the Human Body:307316 doi:DOI 10.1007/978-1-4419-7089-3_15

De Filippis F, Parente E, Ercolini D (2018) Recent Past, Present, and Future of the Food Microbiome. Annu Rev Food Sci Technol 9:589-608 doi:10.1146/annurev-food-030117-012312

DeJong EN, Surette MG, Bowdish DME (2020) The Gut Microbiota and Unhealthy Aging: Disentangling Cause from Consequence. Cell Host Microbe 28(2):180189 doi:10.1016/j.chom.2020.07.013

Deurenberg RH, Bathoorn E, Chlebowicz MA, Couto N, Ferdous M, Garcia-Cobos S, Kooistra-Smid AM, Raangs EC, Rosema S, Veloo AC, Zhou K, Friedrich AW, Rossen JW (2017) Application of next generation sequencing in clinical microbiology and infection prevention. J Biotechnol 243:16-24 doi:10.1016/j.jbiotec.2016.12.022

Ellsworth BA, Tom NJ, Bartlett PA (1996) Synthesis and evaluation of inhibitors of bacterial D-alanine:D-alanine ligases. Chem Biol 3(1):37-44 doi:10.1016/s1074-5521(96)90082-4

Feng G, Xie T, Wang X, Bai J, Tang L, Zhao H, Wei W, Wang M, Zhao Y (2018) Metagenomic analysis of microbial community and function involved in cdcontaminated soil. BMC Microbiol 18(1):11 doi:10.1186/s12866-018-1152-5

Fingas F, Volke D, Hassert R, Fornefett J, Funk S, Baums CG, Hoffmann R (2019) Sensitive and immunogen-specific serological detection of Rodentibacter pneumotropicus infections in mice. BMC Microbiol 19(1):43 doi:10.1186/s12866-019-1417-7

Page $7 / 13$ 
Frank JA, Reich Cl, Sharma S, Weisbaum JS, Wilson BA, Olsen GJ (2008) Critical evaluation of two primers commonly used for amplification of bacterial 16S rRNA genes. Appl Environ Microbiol 74(8):2461-70 doi:10.1128/AEM.02272-07

Freitas AC, Hill JE (2018) Bifidobacteria isolated from vaginal and gut microbiomes are indistinguishable by comparative genomics. PLoS One 13(4):e0196290 doi:10.1371/journal.pone.0196290

Fujiyoshi S, Muto-Fujita A, Maruyama F (2020) Evaluation of PCR conditions for characterizing bacterial communities with full-length 16S rRNA genes using a portable nanopore sequencer. Sci Rep 10(1):12580 doi:10.1038/s41598-020-69450-9

Gao X, Lin H, Revanna K, Dong Q (2017) A Bayesian taxonomic classification method for 16S rRNA gene sequences with improved species-level accuracy. BMC Bioinformatics 18(1):247 doi:10.1186/s12859-017-1670-4

Gevers D, Knight R, Petrosino JF, Huang K, McGuire AL, Birren BW, Nelson KE, White O, Methe BA, Huttenhower C (2012) The Human Microbiome Project: a community resource for the healthy human microbiome. PLoS Biol 10(8):e1001377 doi:10.1371/journal.pbio.1001377

Gomez-Gallego C, Pohl S, Salminen S, De Vos WM, Kneifel W (2016) Akkermansia muciniphila: a novel functional microbe with probiotic properties. Benef Microbes 7(4):571-84 doi:10.3920/BM2016.0009

Gu W, Miller S, Chiu CY (2019) Clinical Metagenomic Next-Generation Sequencing for Pathogen Detection. Annu Rev Pathol 14:319-338 doi:10.1146/annurevpathmechdis-012418-012751

Heiman ML, Greenway FL (2016) A healthy gastrointestinal microbiome is dependent on dietary diversity. Mol Metab 5(5):317-320 doi:10.1016/j.molmet.2016.02.005

Hemmat-Jou MH, Safari-Sinegani AA, Mirzaie-Asl A, Tahmourespour A (2018) Analysis of microbial communities in heavy metals-contaminated soils using the metagenomic approach. Ecotoxicology 27(9):1281-1291 doi:10.1007/s10646-018-1981-x

Jeong J, Yun K, Mun S, Chung WH, Choi SY, Nam YD, Lim MY, Hong CP, Park C, Ahn YJ, Han K (2021) The effect of taxonomic classification by full-length 16 S rRNA sequencing with a synthetic long-read technology. Sci Rep 11(1):1727 doi:10.1038/s41598-020-80826-9

John GK, Mullin GE (2016) The Gut Microbiome and Obesity. Curr Oncol Rep 18(7):45 doi:10.1007/s11912-016-0528-7

Karst SM, Ziels RM, Kirkegaard RH, Sorensen EA, McDonald D, Zhu Q, Knight R, Albertsen M (2021) High-accuracy long-read amplicon sequences using unique molecular identifiers with Nanopore or PacBio sequencing. Nat Methods 18(2):165-169 doi:10.1038/s41592-020-01041-y

Kchouk M. Gibrat JF, Elloumi M (2017) Generations of Sequencing Technologies: From First to Next Generation. Biology and Medicine 9(3):1-8 doi:DOI: $10.4172 / 0974-8369.1000395$

Lawson MR, Ma W, Bellecourt MJ, Artsimovitch I, Martin A, Landick R, Schulten K, Berger JM (2018) Mechanism for the Regulated Control of Bacterial Transcription Termination by a Universal Adaptor Protein. Mol Cell 71(6):911-922 e4 doi:10.1016/j.molcel.2018.07.014

Liang Y, Dong T, Chen M, He L, Wang T, Liu X, Chang H, Mao JH, Hang B, Snijders AM, Xia Y (2020) Systematic Analysis of Impact of Sampling Regions and Storage Methods on Fecal Gut Microbiome and Metabolome Profiles. mSphere 5(1) doi:10.1128/mSphere.00763-19

Liu B, Wang W, Zhu X, Sun X, Xiao J, Li D, Cui Y, Wang C, Shi Y (2018) Response of Gut Microbiota to Dietary Fiber and Metabolic Interaction With SCFAs in Piglets. Front Microbiol 9:2344 doi:10.3389/fmicb.2018.02344

Losso JN (2021) Food Processing, Dysbiosis, Gastrointestinal Inflammatory Diseases, and Antiangiogenic Functional Foods or Beverages. Annu Rev Food Sci Technol 12:235-258 doi:10.1146/annurev-food-062520-090235

Malla MA, Dubey A, Kumar A, Yadav S, Hashem A, Abd Allah EF (2018) Exploring the Human Microbiome: The Potential Future Role of Next-Generation Sequencing in Disease Diagnosis and Treatment. Front Immunol 9:2868 doi:10.3389/fimmu.2018.02868

Markowiak-Kopec P, Slizewska K (2020) The Effect of Probiotics on the Production of Short-Chain Fatty Acids by Human Intestinal Microbiome. Nutrients 12(4) doi:10.3390/nu12041107

Masco L, Vanhoutte T, Temmerman R, Swings J, Huys G (2007) Evaluation of real-time PCR targeting the 16S rRNA and recA genes for the enumeration of bifidobacteria in probiotic products. Int J Food Microbiol 113(3):351-7 doi:10.1016/j.ijfoodmicro.2006.07.021

Metras BN, Holle MJ, Parker VJ, Miller MJ, Swanson KS (2020) Assessment of commercial companion animal kefir products for label accuracy of microbial composition and quantity. J Anim Sci 98(9) doi:10.1093/jas/skaa301

Mohajeri MH, Brummer RJM, Rastall RA, Weersma RK, Harmsen HJM, Faas M, Eggersdorfer M (2018) The role of the microbiome for human health: from basic science to clinical applications. Eur J Nutr 57(Suppl 1):1-14 doi:10.1007/s00394-018-1703-4

Nash AK, Auchtung TA, Wong MC, Smith DP, Gesell JR, Ross MC, Stewart CJ, Metcalf GA, Muzny DM, Gibbs RA, Ajami NJ, Petrosino JF (2017) The gut mycobiome of the Human Microbiome Project healthy cohort. Microbiome 5(1):153 doi:10.1186/s40168-017-0373-4

Page 8/13 
O'Sullivan DJ, Giblin L, McSweeney PL, Sheehan JJ, Cotter PD (2013) Nucleic acid-based approaches to investigate microbial-related cheese quality defects. Front Microbiol 4:1 doi:10.3389/fmicb.2013.00001

Okamura K, Hayashi M, Abe Y, Kono M, Nakajima K, Aoyama Y, Nishigori C, Ishimoto H, Ishimatsu Y, Nakajima M, Hozumi Y, Suzuki T (2019) NGS-based targeted resequencing identified rare subtypes of albinism: Providing accurate molecular diagnosis for Japanese patients with albinism. Pigment Cell Melanoma Res 32(6):848-853 doi:10.1111/pcmr.12800

Park MJ, Park MS, Ji GE (2019) Improvement of electroporation-mediated transformation efficiency for a Bifidobacterium strain to a reproducibly high level. J Microbiol Methods 159:112-119 doi:10.1016/j.mimet.2018.11.019

Pereira RP, Peplies J, Brettar I, Hofle MG (2017) Development of a genus-specific next generation sequencing approach for sensitive and quantitative determination of the Legionella microbiome in freshwater systems. BMC Microbiol 17(1):79 doi:10.1186/s12866-017-0987-5

Ramsey PH (1989) Critical Values for Spearman's Rank Order Correlation. Journal of Educational Statistics 14(3):245-253 doi:doi:10.2307/1165017

Requena T, Burton J, Matsuki T, Munro K, Simon MA, Tanaka R, Watanabe K, Tannock GW (2002) Identification, detection, and enumeration of human bifidobacterium species by PCR targeting the transaldolase gene. Appl Environ Microbiol 68(5):2420-7 doi:10.1128/AEM.68.5.2420-2427.2002

Rim JH, Lee ST, Gee HY, Lee BJ, Choi JR, Park HW, Han SH, Han J (2017) Accuracy of Next-Generation Sequencing for Molecular Diagnosis in Patients With Infantile Nystagmus Syndrome. JAMA Ophthalmol 135(12):1376-1385 doi:10.1001/jamaophthalmol.2017.4859

Rinninella E, Raoul P, Cintoni M, Franceschi F, Miggiano GAD, Gasbarrini A, Mele MC (2019) What is the Healthy Gut Microbiota Composition? A Changing Ecosystem across Age, Environment, Diet, and Diseases. Microorganisms 7(1) doi:10.3390/microorganisms7010014

Russell DA, Ross RP, Fitzgerald GF, Stanton C (2011) Metabolic activities and probiotic potential of bifidobacteria. Int J Food Microbiol 149(1):88-105 doi:10.1016/j.ijfoodmicro.2011.06.003

Schriefer AE, Cliften PF, Hibberd MC, Sawyer C, Brown-Kennerly V, Burcea L, Klotz E, Crosby SD, Gordon JI, Head RD (2018) A multi-amplicon 16S rRNA sequencing and analysis method for improved taxonomic profiling of bacterial communities. J Microbiol Methods 154:6-13 doi:10.1016/j.mimet.2018.09.019

Serafini F, Turroni F, Guglielmetti S, Gioiosa L, Foroni E, Sanghez V, Bartolomucci A, Motherway MO, Palanza P, van Sinderen D, Ventura M (2012) An efficient and reproducible method for transformation of genetically recalcitrant bifidobacteria. FEMS Microbiol Lett 333(2):146-52 doi:10.1111/j.1574$6968.2012 .02605 . x$

Sharifi-Rad J, Rodrigues CF, Stojanovic-Radic Z, Dimitrijevic M, Aleksic A, Neffe-Skocinska K, Zielinska D, Kolozyn-Krajewska D, Salehi B, Milton Prabu S, Schutz F, Docea AO, Martins N, Calina D (2020) Probiotics: Versatile Bioactive Components in Promoting Human Health. Medicina (Kaunas) 56(9) doi:10.3390/medicina56090433

Shehata HR, Ragupathy S, Shanmughanandhan D, Kesanakurti P, Ehlinger TM, Newmaster SG (2019) Guidelines for Validation of Qualitative Real-Time PCR Methods for Molecular Diagnostic Identification of Probiotics. J AOAC Int 102(6):1774-1778 doi:10.5740/jaoacint.18-0320

Shukla RYaP (2017) Probiotics for Human Health: Current Progress and Applications. Recent Advances in Applied Microbiology:133-147 doi:DOI 10.1007/978981-10-5275-0_6

Slatko BE, Gardner AF, Ausubel FM (2018) Overview of Next-Generation Sequencing Technologies. Curr Protoc Mol Biol 122(1):e59 doi:10.1002/cpmb.59

Sperling JL, Silva-Brandao KL, Brandao MM, Lloyd VK, Dang S, Davis CS, Sperling FAH, Magor KE (2017) Comparison of bacterial 16S rRNA variable regions for microbiome surveys of ticks. Ticks Tick Borne Dis 8(4):453-461 doi:10.1016/j.ttbdis.2017.02.002

Stenson PD, Mort M, Ball EV, Evans K, Hayden M, Heywood S, Hussain M, Phillips AD, Cooper DN (2017) The Human Gene Mutation Database: towards a comprehensive repository of inherited mutation data for medical research, genetic diagnosis and next-generation sequencing studies. Hum Genet 136(6):665677 doi:10.1007/s00439-017-1779-6

Theis KR, Romero R, Winters AD, Greenberg JM, Gomez-Lopez N, Alhousseini A, Bieda J, Maymon E, Pacora P, Fettweis JM, Buck GA, Jefferson KK, Strauss JF, 3rd, Erez O, Hassan SS (2019) Does the human placenta delivered at term have a microbiota? Results of cultivation, quantitative real-time PCR, 16S rRNA gene sequencing, and metagenomics. Am J Obstet Gynecol 220(3):267 e1-267 e39 doi:10.1016/j.ajog.2018.10.018

Tran Q, Pham DT, Phan V (2017) Using 16S rRNA gene as marker to detect unknown bacteria in microbial communities. BMC Bioinformatics 18(Suppl 14):499 doi:10.1186/s12859-017-1901-8

Walters W, Hyde ER, Berg-Lyons D, Ackermann G, Humphrey G, Parada A, Gilbert JA, Jansson JK, Caporaso JG, Fuhrman JA, Apprill A, Knight R (2016) Improved Bacterial 16S rRNA Gene (V4 and V4-5) and Fungal Internal Transcribed Spacer Marker Gene Primers for Microbial Community Surveys. mSystems 1(1) doi:10.1128/mSystems.00009-15

Wang C, Zhao J, Zhang H, Lee YK, Zhai Q, Chen W (2020) Roles of intestinal bacteroides in human health and diseases. Crit Rev Food Sci Nutr:1-19 doi:10.1080/10408398.2020.1802695 
Wang X, Mair R, Hatcher C, Theodore MJ, Edmond K, Wu HM, Harcourt BH, Carvalho Mda G, Pimenta F, Nymadawa P, Altantsetseg D, Kirsch M, Satola SW, Cohn A, Messonnier NE, Mayer LW (2011) Detection of bacterial pathogens in Mongolia meningitis surveillance with a new real-time PCR assay to detect Haemophilus influenzae. Int J Med Microbiol 301(4):303-9 doi:10.1016/j.ijmm.2010.11.004

Williams GM, Leary SD, Ajami NJ, Chipper Keating S, Petrosin JF, Hamilton-Shield JP, Gillespie KM (2019) Gut microbiome analysis by post: Evaluation of the optimal method to collect stool samples from infants within a national cohort study. PLoS One 14(6):e0216557 doi:10.1371/journal.pone.0216557

Wu X, Zhang H, Chen J, Shang S, Wei Q, Yan J, Tu X (2016) Comparison of the fecal microbiota of dholes high-throughput Illumina sequencing of the V3-V4 region of the 16S rRNA gene. Appl Microbiol Biotechnol 100(8):3577-86 doi:10.1007/s00253-015-7257-y

Yarza P, Yilmaz P, Pruesse E, Glockner FO, Ludwig W, Schleifer KH, Whitman WB, Euzeby J, Amann R, Rossello-Mora R (2014) Uniting the classification of cultured and uncultured bacteria and archaea using 16S rRNA gene sequences. Nat Rev Microbiol 12(9):635-45 doi:10.1038/nrmicro3330

Young VB (2017) The role of the microbiome in human health and disease: an introduction for clinicians. BMJ 356:j831 doi:10.1136/bmj.j831

Zemb O, Achard CS, Hamelin J, De Almeida ML, Gabinaud B, Cauquil L, Verschuren LMG, Godon JJ (2020) Absolute quantitation of microbes using 16S rRNA gene metabarcoding: A rapid normalization of relative abundances by quantitative PCR targeting a 16S rRNA gene spike-in standard. Microbiologyopen 9(3):e977 doi:10.1002/mbo3.977

\section{Tables}

Table 1. Overall information of bacterial genus-specific primer set

\begin{tabular}{|c|c|c|c|c|c|c|}
\hline Bacterial taxon & Rank & Target gene & Foward primer ( $\left.5^{\prime}-3^{\prime}\right)$ & Reverse primer ( $\left.5^{\prime}-3^{\prime}\right)$ & $\underset{(\mathrm{F} / \mathrm{R})}{{ }^{\circ} \mathrm{C}}$ & $\begin{array}{l}\text { GC } \% \\
\text { (F/R }\end{array}$ \\
\hline Akkermansia & Genus & $d d l$ & CTTCGTGCTGGAAATCAACACC & CGATAATTCCGCTATTTTTTCGC & $62.1 / 59.2$ & $50 / 3 !$ \\
\hline Bacteroides & Genus & $n u s G$ & GGTGCCTCTCAGACAATCAG & CAATGATACCACTGAATCCGCT & $60.5 / 60.1$ & $55 / 4 !$ \\
\hline Bifidobacterium & Genus & Transaldolase & AAGGGCATCTCCGTCAACG & GGAGACGAAGAAGGAAGCGA & $59.5 / 60.5$ & $58 / 5 !$ \\
\hline Phascolarctobacterium & Genus & $n u s G$ & TTCCTGGTTATGTGCTTGTAGAG & CAGTCAAAGGAATCGGTTTAGTA & $60.9 / 59.2$ & $43 / 3 !$ \\
\hline Roseburia & Genus & $n u s G$ & AAATACCCGTGGTGTTACCG & GTGTCTCCСTCTGTAAAGTCA & $58.4 / 59.5$ & $50 / 4 i$ \\
\hline
\end{tabular}

Table 2. Average standard curve calculation results using qRT-PCR assay

\begin{tabular}{lcccc}
\hline Dilution factor & Average ${ }^{*}$ Ct value & *SD value & ${ }^{*}$ CV value & Target gene \\
\hline $10^{\wedge}-3$ from $10 \mathrm{ng}$ & 27.31 & 0.49 & 1.78 & 16S rRNA V4 region \\
$10^{\wedge}-2$ from $10 \mathrm{ng}$ & 23.26 & 0.52 & 2.24 & 16S rRNA V4 region \\
$10^{\wedge}-1$ from 10 ng & 19.85 & 0.64 & 3.21 & 16S rRNA V4 region \\
\hline
\end{tabular}

*Ct value: Cycle threshold value; $*$ SD value: Standard deviation value; $*$ CV value: Coefficient of variation value

Table 3. Statistical result of the Spearman correlation test between two different quantification methods

\begin{tabular}{lccc}
\hline & \multicolumn{3}{c}{ Spearman correlation test } \\
\hline Bacterial genus & $*$ R value & Spearman $P$-value & Spearman's Sig. \\
\hline Akkermansia & 0.89562266 & $2.98 \mathrm{E}-36$ & $* * *$ \\
Bacteroides & 0.62412241 & 0 & $* * *$ \\
Bifidobacterium & 0.8538906 & $1.51 \mathrm{E}-29$ & $* * *$ \\
Phascolarctobacterium & 0.6444568 & $4.67 \mathrm{E}-13$ & $* * *$ \\
Roseburia & 0.51864254 & $3.25 \mathrm{E}-08$ & $* * *$ \\
\hline
\end{tabular}

*Sig.: Statistical significance; *R: Spearman's rho value 
Table 4. Bacterial identification result by Sanger sequencing

\begin{tabular}{|c|c|c|c|c|c|c|}
\hline \multirow[b]{2}{*}{ Bacterial genus } & \multicolumn{3}{|c|}{ Defined bacrterial taxon counts in NCBI database } & \multicolumn{3}{|c|}{ Defined bacterial taxon rates (\%) of Sanger validation } \\
\hline & $\begin{array}{c}\text { Major Top } 5 \text { (Ct } \\
\text { value) }\end{array}$ & $\begin{array}{c}\text { Minor Top5 (Ct } \\
\text { value) }\end{array}$ & Total & $\begin{array}{c}\text { Major Top } 5 \text { (Ct } \\
\text { value) }\end{array}$ & $\begin{array}{c}\text { Minor Top5 (Ct } \\
\text { value) }\end{array}$ & Total \\
\hline Akkermansia & 25 & 25 & 50 & 100.00 & 100,00 & 100.00 \\
\hline Bacteroides & 25 & 24 & 49 & 100.00 & 96.00 & 98.00 \\
\hline Bifidobacterium & 23 & 15 & 38 & 92.00 & 60.00 & 76.00 \\
\hline Phascolarctobacterium & 25 & 25 & 50 & 100.00 & 100.00 & 100.00 \\
\hline Roseburiea & 25 & 25 & 50 & 100.00 & 100.00 & 100.00 \\
\hline
\end{tabular}

\section{Figures}

a

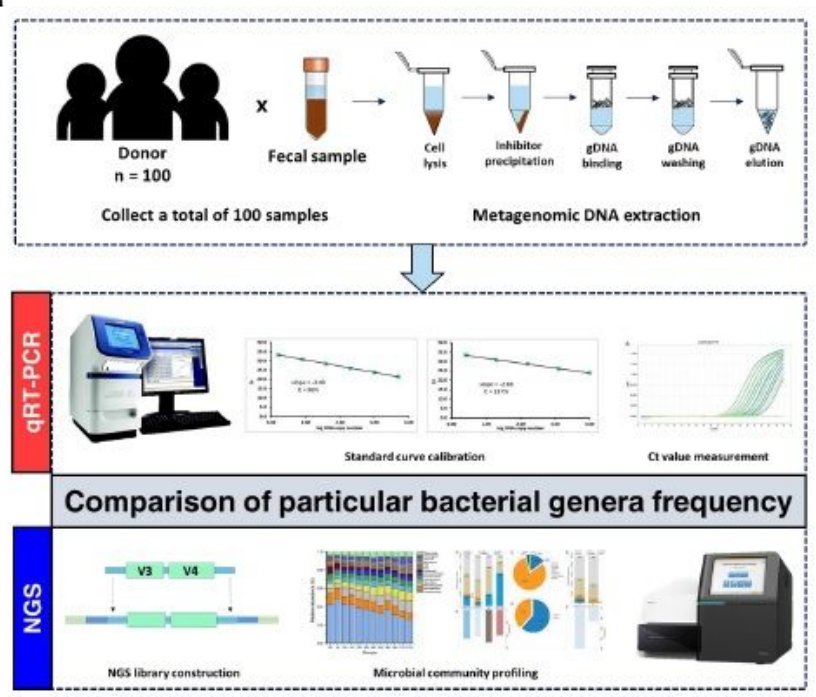

b

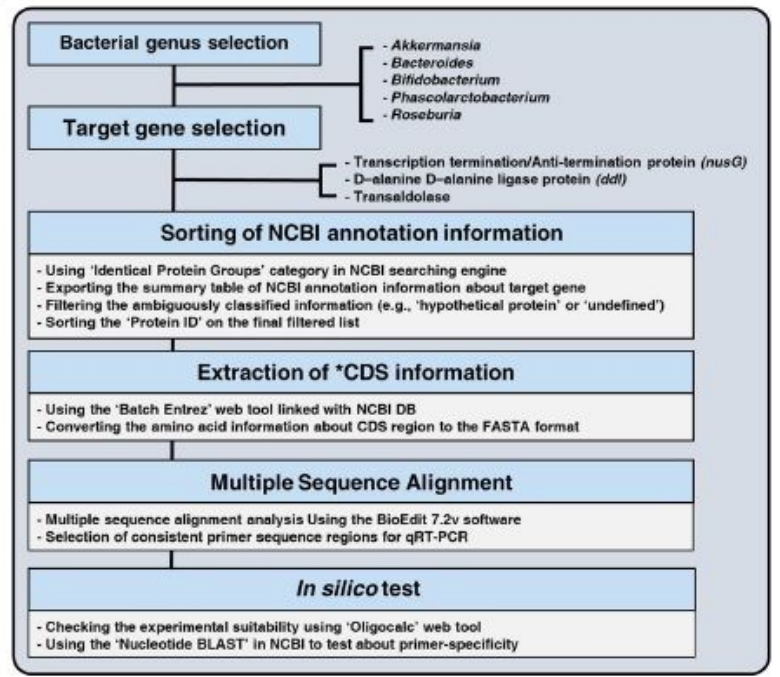

Figure 1

Experimental introduction in this study and schematic workflow of genus-specific primer design method for qRT-PCR assay. Using stool samples obtained from 100 healthy adults, we performed a comparative analysis to evaluate the accuracy of microbial quantification by two different molecular technologies.

(a) The overall experimental workflow of the qRT-PCR assay and NGS-based 16S V3-V4 metagenome sequencing. (b) Schematic diagram showing the process to design the genus-specific primer set to quantify the proportions of the five selected bacterial genera within each sample using qRT-PCR.

a

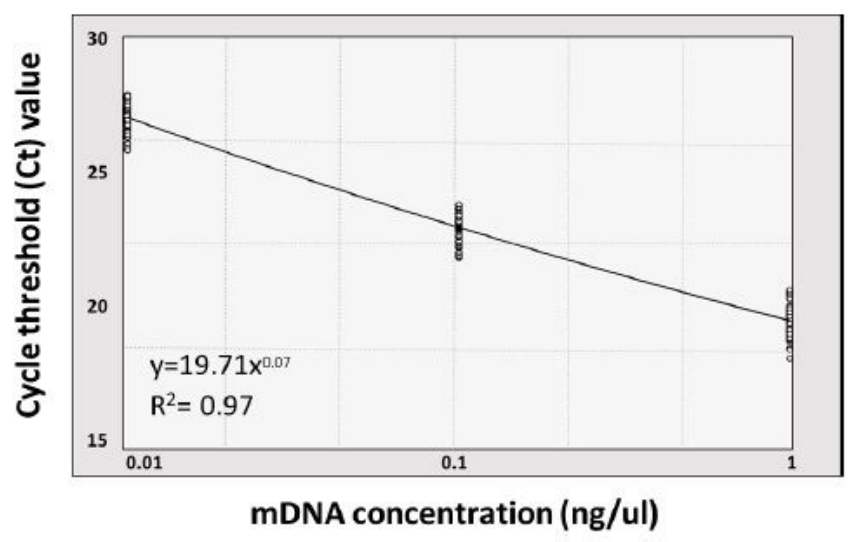

b

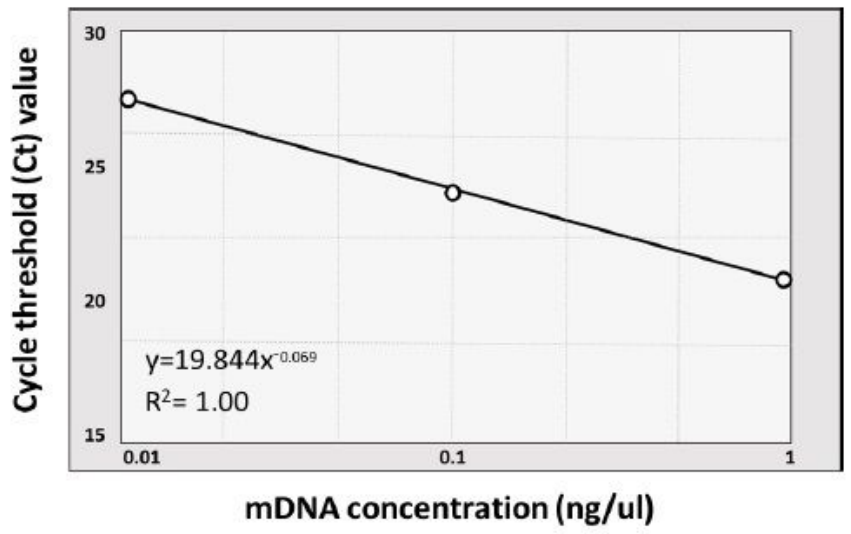


Figure 2

Standard curve calculation to confirm normalization of the mDNA concentration used for qRT-PCR analysis. Each double-stranded mDNA sample diluted with a three step 10-fold serial dilution (10-1, 10-2, and 10-3) was standardized at $10 \mathrm{ng} / \mathrm{ul}$ for standard curve calculation. The graph's x-axis indicates the concentration of each 10-fold diluted mDNA used in standard curve calculation, and the y-axis shows the Ct value measured from the qRT-PCR. (a) Standard curve graph representing the $\mathrm{Ct}$ value of every samples with 10 -fold serial diluted mDNA concentration (R2 $=0.97)$. (b) Standard curve graph representing the average $\mathrm{Ct}$ value of each concentration of the diluted mDNA sample $(\mathrm{R} 2=1.00)$.
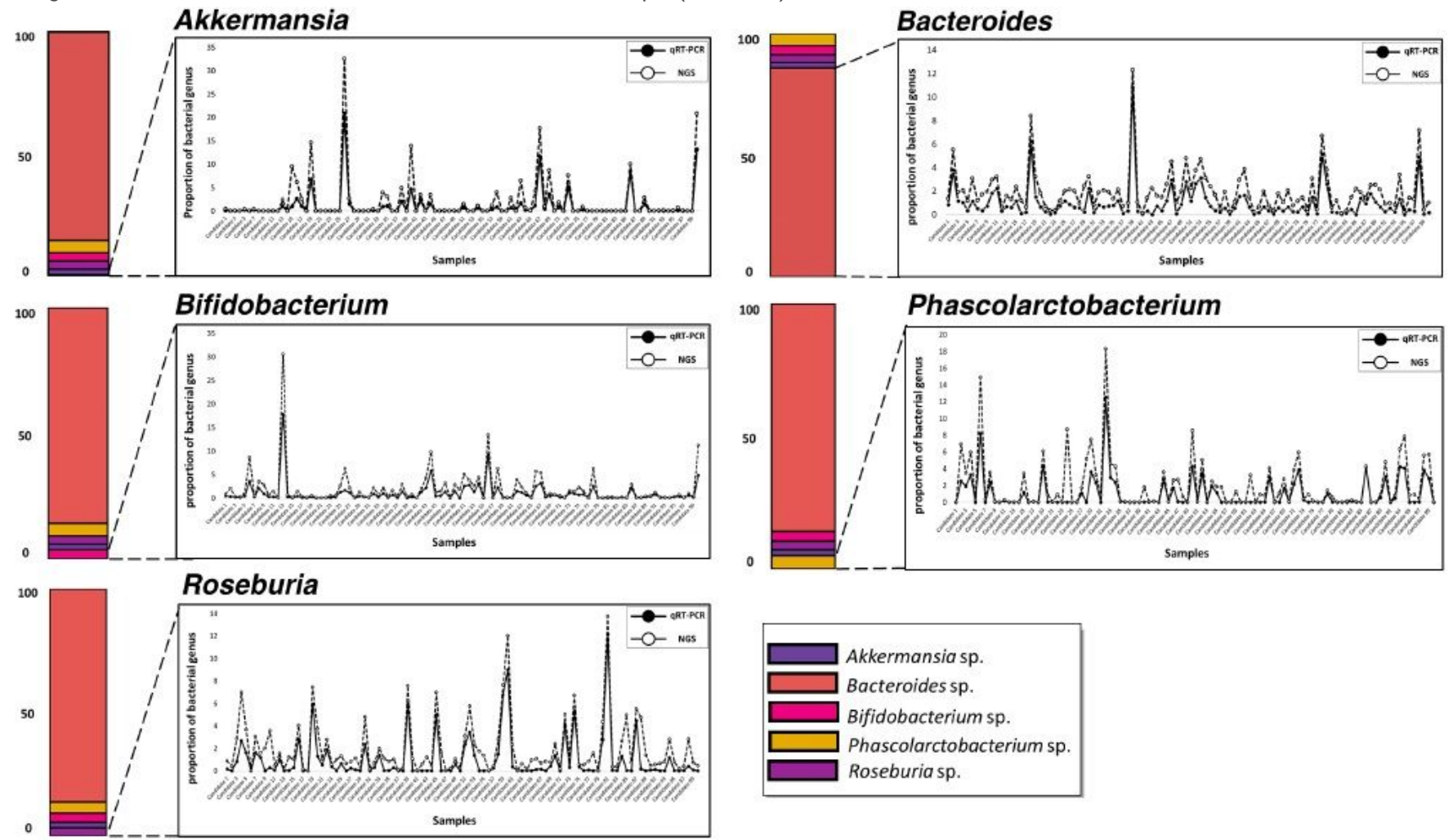

Figure 3

Parallel comparison of the interest five bacterial genera proportions measured from two different quantification methods. Multiple overlaid connected line graphs show the quantitative identity of each bacterial genus measured by both methods. The graph's $\mathrm{x}$-axis indicates the 100 samples (denote as 'candidate'), and the $y$-axis indicates the relative proportion value for each particular bacterial genus. The bar plot shown on the left side represents an average abundance of five bacterial genera calculated from $16 \mathrm{~S}$ metagenomic profiling analysis.

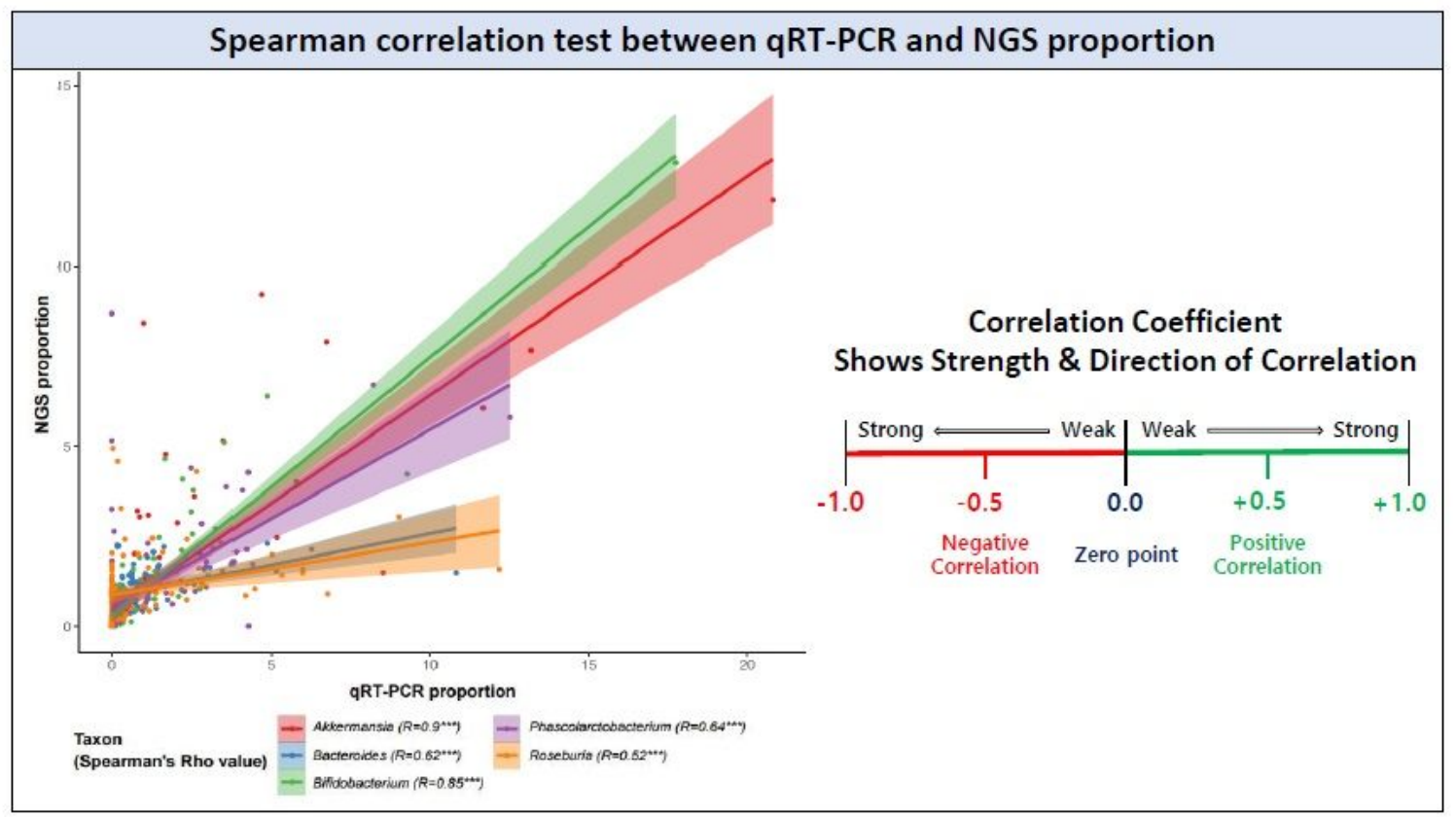


Figure 4

Spearman correlation scatter plot showing the relationship of each bacterial relative proportion value measured by qRT-PCR assay and 16S V3-V4 metagenome sequencing data; $* * *=$ Spearman P-value $<0.001, \mathrm{R}=$ Spearman's rho value.

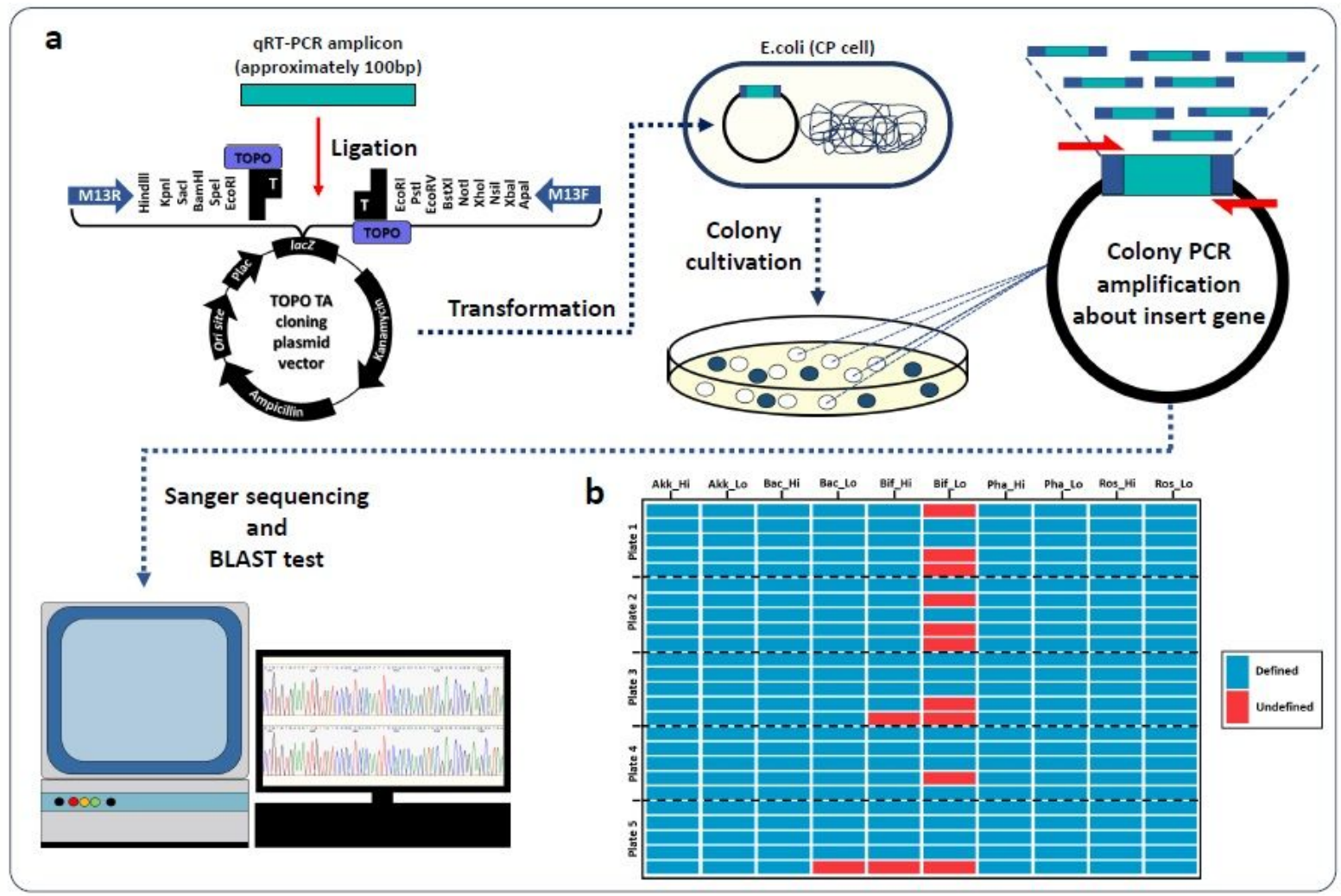

Figure 5

Verification of the specificity and accuracy of primers for quantifying the five bacteria genera using Sanger sequencing. (a) The schematic diagram shows the overall experimental process for TA cloning of each genus-specific amplicon, bacterial gene transformation, Sanger sequencing validation, and sequence confirmation through NCBI nucleotide BLAST search. (b) The confirmation of the nucleotide sequence of the genus-specific amplicons, showing five high and low frequencies, respectively, is shown in the mosaic plot. The sky-blue and red colors denote the BLAST test identified the appropriate target bacterial genus name and not working, respectively.

\section{Supplementary Files}

This is a list of supplementary files associated with this preprint. Click to download.

- Sangervalidataionfile.egg

- SupplementalFig.S1.pdf

- SupplementalTableS1.xlsx

- SupplementalTableS2.xlsx

- SupplementalTableS3.xlsx

- SupplementalTableS4..xlsx

- SupplementalTableS5.xlsx

- SupplementalTableS6.xIsx

- SupplementalTableS7.xlsx 